\title{
Paper-trail index
}

\author{
Can a metric that captures a paper's history of submission and rejection give insight into its value \\ and encourage good authorship practices?
}

\author{
Shai Berlin
}

$\mathrm{H}$ ow many scientific manuscripts are submitted first to high-ranking publications, such as Nature or Science, and then wind their way down a list of decreasingly prestigious journals until they eventually get accepted? There is currently no way to find out how many stops a scientific paper has made along its way to publication — or into oblivion - but there is a general feeling among scientists, reviewers, and editors that a considerable number of manuscripts probably have an extensive paper trail. Many papers follow such a winding and convoluted path to publication because scientists are both under pressure and aspire to publish in the most prestigious journals with the highest impact factors (IF). This has created a hypercompetitive environment in which the IF of the journal a paper is published in has become more important than the quality of a paper itself. In turn, this has reinforced the trend of trying to get all papers published in high-ranking journals, regardless of whether the authors genuinely believe such a journal is appropriate for their manuscript, or whether their manuscript is appropriate for such a journal. Sadly, this pressure to publish in top journals also contributes to scientific misconduct [1]. This merry-go-round of submission, rejection, revision, and resubmission has led to a colossal number of papers in circulation [2] - 12,000 papers submitted a year to Science [3], 11,000/year to Nature (http://www.nature. com/nature/authors/get_published/), and 5,000/year to the New England Journal of Medicine (http://www.nejm.org/page/mediacenter/publication-process) — of which only a small percentage are sent to review and an even smaller percentage are published in the journals to which they were originally sent. This flood of articles is clogging the publishing system, and unnecessarily occupying the time of reviewers and editors alike.

The question is whether the paper trail of any given manuscript can tell us anything about its actual merits. For example, if a paper was immediately accepted for publication at the first place it was sent to can we infer that the manuscript was already well developed, the findings novel, and relevant, the experiments properly designed and the results properly interpreted? Or does it mean that the authors "aimed low" if the journal was not one of the high-IF publications? In that vein, is "aiming low" a bad thing, or does it simply show that the authors had a good sense of the novelty and value of their work and the size of their audience; and thus submitted it to a suitable journal, perhaps one more deeply embedded in a specialized and relevant research community? Inversely, do multiple or serial submissions mean that a paper lacks clear, welldone, novel findings, or does it just mean that the authors repeatedly "aimed too high" to try to get published in a high-IF journal? And if so, should this be penalized as a waste of everyone's time?

T he answers to the questions above are likely to be nuanced and hard to capture. Nevertheless, such a metricperhaps a "paper-trail index", or PTI value - that formally and comparably describes the number of submissions a paper has undergone prior to publication and the main reasons why it was not accepted for review or publication at each stage might add significant value to the range of metrics we already use to assess the value of the research. It would also provide benefits above and beyond the current range of metrics in use: the PTI would mean that the time currently "wasted" assessing papers at journals where they are not accepted is acknowledged and rated when a paper is finally published; it would increase transparency in publishing, both about the history of a paper and about individual journals' review processes and acceptance criteria; it might reduce the number of papers that spiral downwards through the publication system that potentially waste time and resources; and, importantly, it would provide an alternative measure of the quality of a paper itself, if it summarizes the views of editors and referees who had seen the paper, rather than just the editor and referees who finally agreed to publish it.

cc

"... there is a general feeling

among scientists, reviewers

and editors that a considerable number of manuscripts probably have an extensive paper trail”

As noted above, capturing all of this information in a single score may, at first, prove to be extremely difficult. As such, a PTI score might best be viewed in conjunction with all the information behind it, rather than as a stand-alone value. Nevertheless, there would still be use in the PTI score alone as an additional metric by which to assess an individual scientist's publication record. The index would examine the number of submissions and score negative and positive comments, by editors and reviewers, and compile the data into one 


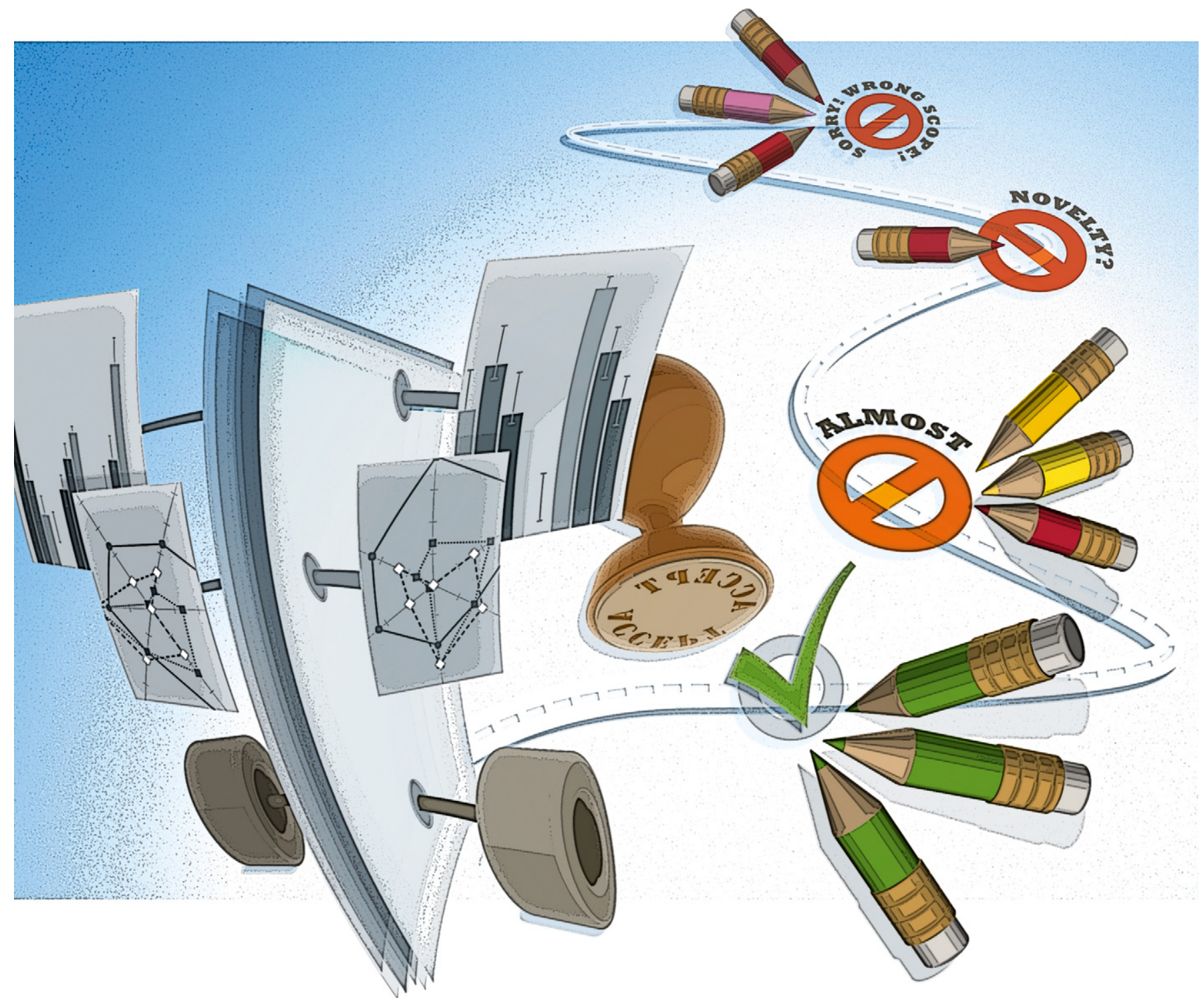

comprehensive score. This would, in particular, contrast the IF, as it portrays the paper rather than the journal.

To efficiently score a paper's assessment at a given journal, it should be sufficient to rate the referee's general remarks and the editor's decision letter, rather than attempting to analyze the detailed referee reports, which can be several pages long. First, these to-the-point comments distill and emphasize the main points of concern, whereas the detailed review might include a variety of less pertinent remarks about languages, mistakes in references, color usage, swapped legends, etc. Second, general remarks allow non-experts in the field to quickly grasp what the paper was missing. Third, it alleviates the need to read the detailed reviews and subjectively score them. This point would be particularly crucial for an objective and automated scoring process.

A part from evaluating a paper's value and track record, the PTI might have a positive effect on the publication process itself. It might encourage authors to be more realistic in deciding where to submit their papers, perhaps avoiding hail-Mary passes to high-IF journals that are unlikely to accept them, as this will directly impact on the paper's PTI. This could reduce the number of submissions to highly sought-after journals, increase the number of good submissions to other journals, and reduce the total number of papers in circulation limbo. This ought to ease the burden on reviewers and editors, which would benefit authors by both reducing decision-making time and potentially increasing the quality of peer review.

Indirectly, as authors become more careful when choosing a journal to submit to, it could also reduce possible "celebrity" bias. Currently, submitting a paper to as many journals as possible does not carry any negative consequences - aside time wasted-and renowned principal investigators (PI) or scientists from prestigious institutions may therefore try to gamble on their famous name and submit to top-tier journals, hoping that editors and reviewers would be more in favor of publishing their paper [4]. While a PTI will not be able to eliminate such bias, it might still reduce it. Researchers who persistently submit their papers to high-ranking journals would incur a penalty, as it will 
negatively affect the score of the manuscript if it does not get accepted.

\section{(c) ... many papers that spiral downwards through submissions to multiple journals do so because their authors have unrealistic aspirations for their research"}

Indirectly, as a result of fewer submissions, the PTI might also reduce the amount of papers that languish for years before they are finally published. Many research projects pass from one graduate student to the next because the group leader wants to publish in a higher-ranking journal and needs to accumulate more data. As a consequence, the first student who laid the groundwork or designed the crucial experiments ends up being just one author among many on the byline, or might even leave the laboratory without any publication. If a PTI rewards authors for directly submitting their work to more specialized journals, then researchers might be compelled to send their papers out earlier once a project has been deemed finished and has generated solid results, rather than expanding the work for years to eventually qualify for a high-IF journal. Of course, there are also papers that take years to publish because the data is simply insufficient or not compelling, or the findings are not novel. However, many papers that spiral downwards through submissions to multiple journals do so because their authors have unrealistic aspirations for their research.

$\mathrm{T}$ he PTI could also be used to determine trends in science. Whereas positive trends can be easily analyzed -by just looking at what is frequently published-negative trends are harder to discern. Some may argue that it would mean simply examining what is published and where, with the notion that trendy is in the top and less trendy in lower-IF journals. However, this is not true (http:// news.sciencemag.org/scientific-community/ 2014/10/uprising-less-prestigious-journalspublishing-greater-share-high-impact). In fact, authors may send their papers to lower-IF journals because these represent highly specialized fields or because these journals do not assess for novelty and broad interest, such as PLoS One. Negative trend analysis can be helpful for authors when they ponder which journal is most appropriate (or not) for their manuscript, but also for specific scientific communities or research funding agencies to inspect whether journals are inadvertently biased toward certain fields of study.

The data behind a PTI could also be used for quality control purposes, by evaluating valuable parameters such as rejection statistics, biases, the interval from one submission to the next and to publication, the time for review, and decision for each journal, whether the index influences reading habits, or whether it affects careers. In fact, given the increasing importance of transparency in scientific publishing, it is odd that these data are not already available in a complete and meaningful way to the research community.

Lastly, a paper-trail index should help to resolve disputes about novelty. Although many journals provide data about when a paper was submitted, reviewed, accepted, and published, these are only visible, and therefore relevant, once the paper has been accepted for publication. What happens when a paper is rejected and moves on to another journal but something similar is published in the meantime? Being "scooped" not only makes it more difficult for a group to publish their findings in a good journal, but also to claim novelty. A PTI detailing the chronology of a publication could therefore help to establish claims of novelty, or even become useful for legal disputes or Intellectual Property claims.

$\mathrm{n}$ n summary, a paper-trail index would clearly provide tangible benefits for the scientific community beyond the currently established indices. However, the increased transparency could also cause some problems and complications. Opponents might claim that there is no correlation between a paper's value and the number of submissions and reviews it had to undergo before it was published. This can be true, but similar criticisms also hold true for the IF or the $h$-index: there is not always correlation between the value of a paper and the IF of the journal it is published in or the number of times it is cited (especially against competing work in higher-IF journals). However, given that the decision to reject or accept a paper is based on expert review by editors or referees, their assessment and critique has merit toward evaluating individual papers. At present, the value of these comments is completely lost. Moreover, many papers that have been submitted multiple times before acceptance have often been improved along the way, based on the reviewers' comments [2]. Thus, the track record of a paper can provide meaningful information about its value and improvement.

Another concern is that a PTI could introduce bias into the peer-review process [4]: editors or reviewers might be inclined to dismiss a paper based on its previous submissions, rather than its content. It is hard to tell whether this will happen, just as it is yet not clear whether other changes, such as double-blind review, would decrease bias [5]. But it is not very likely that all reviewers would be biased. Some reviewers might be even more positive if the authors have considerably improved their work taking into consideration previous critiques, or scrutinize a paper more carefully to understand why it was previously rejected.

\section{"The question is whether the paper trail of any given manuscript can tell us anything about its actual merits"}

At present, some journals and publishers have already begun to share reviewers' comments when transferring papers between journals. This "peer-review transfer," used by BioMed Central (BMC) publishing group, EMBO Press, Nature Publishing Group, and others, applies when a rejected paper is resent to another journal within each consortium - and sometimes between them-to help shorten its time to publication. BMC states that this has been so successful that they have expanded the consortium to include other journals (http:// blogs.biomedcentral.com/bmcseriesblog/ 2013/06/11/portable-peer-review-to-preventa-pillar-to-post-process/). Given the positive experience with transferring reviews, it should not present major concerns or introduce bias, but actually reduce the time to publication. Yet, as for many other changes of the peer-review system, only time will tell 
if it benefits authors and the scientific community. If it did prove to be a serious problem, referees, and editors could be blinded to the PTI until they were done assessing the paper.

A nother hurdle is that researchers might be reluctant to share reviewers' verdicts of their manuscript, fearing it might badly reflect on the group's reputation. This should not really discourage authors, however, as many scientists already post entire manuscripts prior to publication in bioRxiv and other pre-publication depositories to gain critical feedback from their peers and further improve their work before they formally submit it to a journal. So far, this has neither negatively affected the reputation of authors, nor has it jeopardized their chance to get accepted for publication: many high-impact journals now accept submissions of papers that were previously posted on pre-submission sites [6]. In some ways, pre-publication depositories already achieve some of the advantages offered by the PTI: they establish novelty, reduce the risk of being "scooped," and reduce the amount of papers in circulation as authors wait for feedback from their peers before sending their paper out to peer review. Yet, posting manuscripts on pre-publication servers is entirely voluntary. Thus, to introduce a PTI and alleviate any concerns, journals willing to partake in this endeavor could ask authors during submission whether they are willing to disclose information about previous submissions and the main critiques once it is published.

\section{"... a paper-trail index should help to resolve disputes about novelty"}

Journals might also be hesitant to share this information, but I cannot see any reason why they should not. The PTI is not likely to affect a journal's IF-indeed, it should ensure that journals receive fewer inappropriate submissions. Also, the more that research groups become familiar and comfortable with sharing this type of information-even demanding it-the more likely it is that journals will follow-suit and make it available too.
Indeed, a formalized database to which all papers are registered and all journals contribute would be the ideal solution, but might also be the greatest hurdle. A central, open database to track papers through the publication process, analyze their performance and reviews, provide this information in an accessible and easy-to-use way, and calculate their ranking would be a major undertaking. The website would need to provide a unique identifier to each manuscript, which would include information that is likely to remain unchanged, such as name and institution of the first and senior author, subject and title of the paper, date of entry, and so on. Authors could either register their paper with the central website to receive an identifier, or a journal could register the paper on behalf of the authors the first time it is submitted. At each resubmission, the authors would either submit the identifier along with the manuscript, or the journals would need some way of querying the database for similar submissions. Journals in turn would then send their decision summary to the central server, including reviewers' general comments. This information could then be used to automatically calculate an index-rather than relying on human judgment-that would dynamically change with each resubmission, but also to provide the more nuanced data behind the metric. A more sophisticated alternative would be to upload papers directly to a central website and then choose a journal. The website would automatically issue a sort of passport and then redirect the paper to the chosen journal's submission system.

There is certainly a risk that authors might try to game the system by resubmitting papers that have thus-far performed badly with one identifier in order to start anew with a clean paper trail. But it should be rather simple to compare new submissions with existing papers that have not yet been published. If the same or a highly similar entry (same first and senior author or similar title) is found, journals could refuse to accept the paper for submission. Nonetheless, since the whole publication process is based on trust, which generally works fairly well, only a minority of researchers might abuse the system, in the same way that a minority will abuse any system. Occasionally, there might even be justification for formally issuing a new identifier if the research and the revised paper has significant new findings, a different first author or significantly changed conclusions after a few rounds of submission and rejection. In such cases, authors would need to justify how the second paper is sufficiently different when they formally apply for a new identifier.

$\mathrm{n}$ the age of petabytes and exabytes of scientific information, establishing such a database to capture the majority of submissions should not present a major technical problem. PubMed, for example, already collects most of the biomedical literature and is extremely plastic: it has many copy sites, incorporates external extensions, and so on. Moreover, many publishers and journal consortia already have their own databases that store extensive information about submitted, rejected, and accepted papers along with reviews and editorial comments. The biggest hurdle might be to convince publishers to participate in such a "manuscript passport" system and submit standardized information about their decisions and reviewer comments. However, this problem should not be insurmountable. Most journals readily submit information about accepted papers to PubMed, while some publishersnotably EMBO Press and eLife-already publish the full referee reports alongside accepted papers. As their experience with this "transparent review" has been widely regarded as positive, it should help to convince publishers to share data on reviews and decisions as part of a paper-trail index.

\section{"If done well, the PTI could become an alternative to the IF or the $h$-index in assessing research quality or the performance of individual researchers or research groups"}

If done well, the PTI could become an alternative to the IF or the $h$-index in assessing research quality or the performance of individual researchers or research groups, as it focuses on individual papers rather than the quality of journals or a scientist's total publication output. Yet, it would have to meet several criteria to do 
so: reliability-it would have to offer a genuine measure of a paper's value, capturing all of the nuance and case-specific events in a paper's complex history, ease of use-the score and information should provide a quick and easily understandable overview of a paper's history, and resistance to abuse. The index should also be accessible for all involved in the publishing process, from authors to journals to reviewers, research institutions, and funding agencies.

The biggest complication is to start the ball rolling. To this end, all that may be needed is a single journal or publisher to start the experiment on a small scale with the help of bibliometricians to work out how to specifically calculate a nuanced and meaningful score for each paper. In addition, authors could also be encouraged to provide information about the fate of their papers, irrespective of whether their publishing journal participates or not. Yet, if done properly, a paper-trail index could not only drastically increase transparency in scientific publishing, but could ultimately provide a viable alternative or even replace the ill-fated IF and thereby ease the pressure on scientists to publish or perish.

\section{Conflict of interest}

The author declares that he has no conflict of interest.

\section{References}

1. Fang FC, Casadevall A (2011) Retracted science and the retraction index. Infect Immun 79: $3855-3859$

2. Jennings CG (2006) Quality and value: the true purpose of peer review. Nature doi:10.1038/ nature05032.

3. Kelner K (2007) Tips for Publishing in Scientific Journals. Science doi:10.1126/science.caredit. a0700046

4. Smith R (2006) Peer review: a flawed process at the heart of science and journals. J $R$ Soc Med 99: 178-182

5. Editorial (2015) Nature journals offer doubleblind review. Nature 518: 274

6. Editorial (2005) Nature respects preprint servers. Nature 434: 257 